\title{
Cell counting for in vivo flow cytometry signals with baseline drift
}

\author{
Xiaoling Wang*, Yuanzhen Suo*, Dan Wei*, Hao He*, \\ Fan $\mathrm{Wu}^{\dagger, \S}$ and Xunbin Wei** \\ *Med-X Research Institute and School of Biomedical Engineering \\ Shanghai Jiao Tong University, 1954 Huashan Road \\ Shanghai 200030, P. R. China \\ ${ }^{\dagger}$ School of Naval Architecture, Ocean and Civil Engineering \\ Shanghai Jiao Tong University, 800 Dongchuan Road \\ Shanghai 200240, P. R. China \\ *xwei01@sjtu.edu.cn \\ §anwu@sjtu.edu.cn
}

Received 15 May 2016

Accepted 24 January 2017

Published 20 March 2017

\begin{abstract}
In biomedical research fields, the in vivo flow cytometry (IVFC) is a widely used technology which is able to monitor target cells dynamically in living animals. Although the setup of IVFC system has been well established, baseline drift is still a challenge in the process of quantifying circulating cells. Previous methods, i.e., the dynamic peak picking method, counted cells by setting a static threshold without considering the baseline drift, leading to an inaccurate cell quantification. Here, we developed a method of cell counting for IVFC data with baseline drift by interpolation fitting, automatic segmentation and wavelet-based denoising. We demonstrated its performance for IVFC signals with three types of representative baseline drift. Compared with non-baseline-correction methods, this method showed a higher sensitivity and specificity, as well as a better result in the Pearson's correlation coefficient and the mean-squared error (MSE).
\end{abstract}

Keywords: In vivo flow cytometry; cell counting; baseline drift; signal processing.

\section{Introduction}

In the past decades, there has been a significant step forward in the development of in vivo methods for monitoring circulating cells. Among them, the in vivo flow cytometry (IVFC) is a powerful technique that provides real-time detection and quantitative study of target cells in circulation in live animal models. ${ }^{1-6}$ In the fluorescence-based IVFC,

\$Corresponding author.

This is an Open Access article published by World Scientific Publishing Company. It is distributed under the terms of the Creative Commons Attribution 4.0 (CC-BY) License. Further distribution of this work is permitted, provided the original work is properly cited. 


\section{Wang et al.}

as a fluorescently-labeled cell passes through a focused laser slit which is set across the blood vessel, the fluorescence is excited and can be detected. No blood drawing or sacrifice of animals is needed during this process. Substantial experiments have presented its broad applications in research areas such as cancer metastasis, ${ }^{7-11}$ leukemia, ${ }^{10}$ apoptosis, ${ }^{12}$ immunology. ${ }^{13,14}$ and other biomedical applications. ${ }^{15-17}$ Combined with nanotechnology, the specificity of cancer diagnostics has been greatly improved. ${ }^{18-22}$

As the setup of IVFC has been well established, corresponding signal processing approaches have also been explored to study the signal features and improve the cell counting procedure. ${ }^{23-25}$ Usually, the IVFC data are recorded and processed with inhouse software. As the peaks in IVFC signals represent circulating cells, it is a crucial step to accurately count the peak numbers. A previously used method to count cell peak numbers is called "linegating" method, ${ }^{4}$ in which a line drawn manually is needed to separate dots which represent cells from background signals. Apparently, this "line-gating" method consumes both time and manpower, with a relatively low efficiency and accuracy, thus it is replaced by the automatic threshold approach combined with a dynamic peak picking procedure. ${ }^{26}$ Once the peak reaches beyond the threshold given by a formula, it would be regarded as a cell. This method does not require any control experiments and proves to be an effective method in automatic cell peak counting of the IVFC signal.

Due to animals' respiratory movement, limb movement and photobleaching of tissue autofluorescence, the problem of baseline drift exists in many IVFC signals. A cell peak is identified if the intensity of the peak is above a static threshold, which is related to the baseline level according to previous methods. ${ }^{24-26}$ If IVFC signals with baseline drift are analyzed automatically using the abovementioned automatic threshold algorithm, the output results would be highly inaccurate. For example, if the baseline keeps decreasing over time, cell peaks could not be identified in the late part of the signals if their intensities are not high enough. Since IVFC data from a single experimental animal are usually continuous in a long term, the baseline drift in a time point may ruin the total signal. Thus, new methods are needed to count cells in IVFC signals with baseline drift. The problem of baseline drift also exists in many other fields, such as the electrocardiogram, and corresponding methods have been explored to tackle this problem. ${ }^{27-31}$ Here, we developed an effective processing method to solve baseline drift problem in IVFC signals, which could determine whether to smooth the baseline, divide signal into parts and analyze them separately. Compared with previous algorithms, this new method could remove baseline drift in IVFC signals automatically and shows higher accuracy of cell counting.

\section{Methods}

\subsection{IVFC data}

Raw IVFC data in this work were from our previous work, including IVFC data of studying macrophages and prostate cancer cells,${ }^{14}$ hepatocellular carcinoma cells, ${ }^{11,26}$ optical clearing, ${ }^{25}$ near infrared IVFC data of tracking bone marrow cells and mesenchymal stem cells. ${ }^{24}$

\subsection{IVFC signal analysis}

A representative IVFC signal is shown in Fig. 1, where $X$-axis stands for the acquisition time of signals, and $Y$-axis stands for the voltage value that linearly reflects the fluorescence intensity. In the IVFC signals, the low intensity background is called the baseline, while the high intensity pulse is defined as the peak. The baseline level is associated with certain predictable factors, including the auto-fluorescence from tissues, reflection and scattering of the laser and electrical noise. Each peak corresponds to a fluorescently-labeled cell or cell cluster that is excited by the laser beam as it flows through the focused laser slit across the vessel. In the peak, height reflects the fluorescence intensity emitted from target cells, and peak width shows the time of one cell flowing through the focused laser beam.

\subsection{Baseline correction methods}

Generally, baseline drift in IVFC signals could be categorized into three types. The first type is noise bandwidth change (Fig. 2(a)), where the width of the noise band increases or decreases prominently from a certain point. This problem is related to the change of focal plane due to inconspicuous hyperspasmia, which could be solved in the post-processing procedure by analyzing the signal separately. 


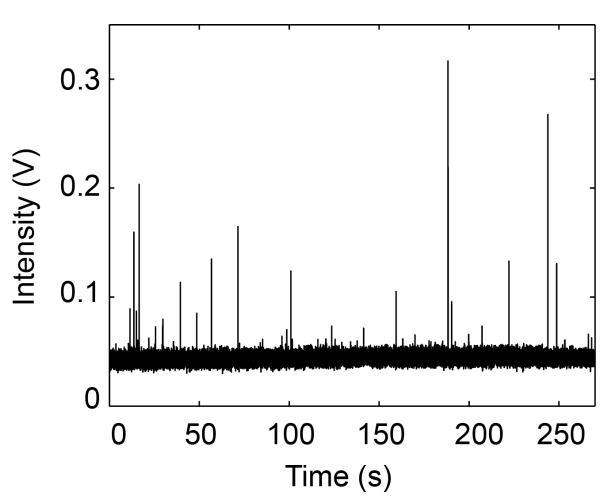

(a)

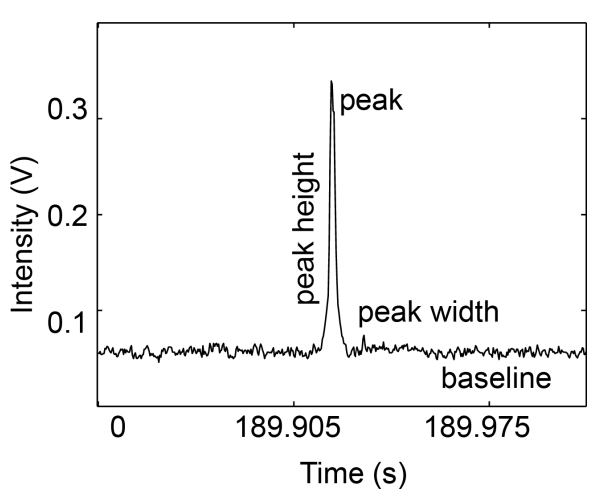

(b)

Fig. 1. Graphic signals of IVFC. (a) A representative IVFC signal. (b) The representative fluorescence signal profile of a cell.

By dividing the signal into several parts according to the width of noise band, each part of the signal with the same noise band width will be given a calculated threshold for peak picking. The second type of baseline drifting is a gentle baseline fluctuation (Fig. 2(b)), in which the signal baseline fluctuates slightly around the average baseline level. This is caused mainly by the animal's normal respiratory movement and photobleaching of tissue auto-fluorescence. The third type is abrupt baseline change, where the baseline level drifts up and down suddenly due to the change of detection site (Fig. 2(c)). By interpolation fitting and subtracting the original signal with the estimated baseline, the baseline could be dragged to a less fluctuating level, thus the problem of gentle baseline fluctuation and abrupt baseline change could be solved at the same time.

The correction method of baseline mainly involves three steps. The first step is interpolation and fitting, through which we can get an estimated baseline of the original signal. A less fluctuating signal could be obtained by subtracting the signal intensity with baseline intensity. Secondly, by checking the intensity difference in the baseline level, the algorithm will automatically decide whether or not to segment the signal. If the signal does not have any problem of intensity difference, the algorithm will go to the final step and output cell counting results. Otherwise, it will segment the signal into parts. The final step is to output cell peak numbers, involving wavelet-based denoising, peak picking and results displaying. Flow chart of the algorithm is shown in Fig. 3.

\subsubsection{Interpolation and fitting}

The aim of the first step is to correct the fluctuating baseline. By setting window size and step size, a series of points would be picked as sampling points. The window size and step size are both set as two points. The interpolation method is Piecewise Cubic Hermite Interpolating Polynomial (PCHIP), which uses a cubic Hermite interpolation and performs

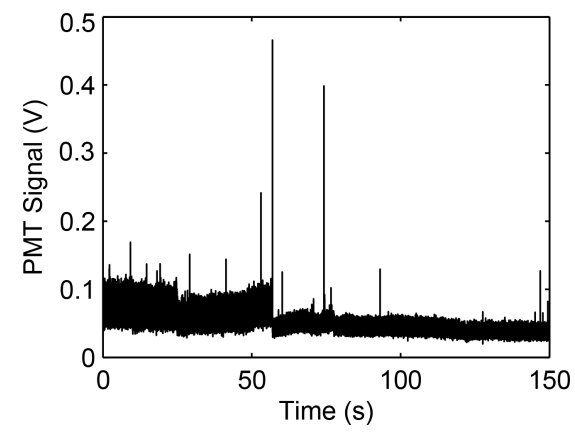

(a)

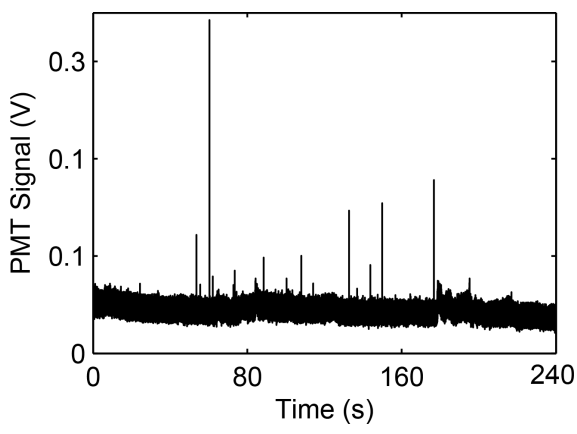

(b)

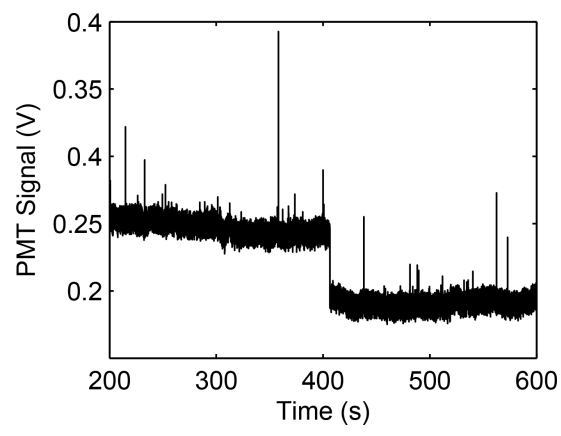

(c)

Fig. 2. Representative IVFC signals with baseline drift. (a) A signal with noise bandwidth change since $57 \mathrm{~s}$. (b) A signal has the problem of gentle baseline fluctuation. (c) An abrupt baseline change occurred at $406 \mathrm{~s}$ in the signal. 


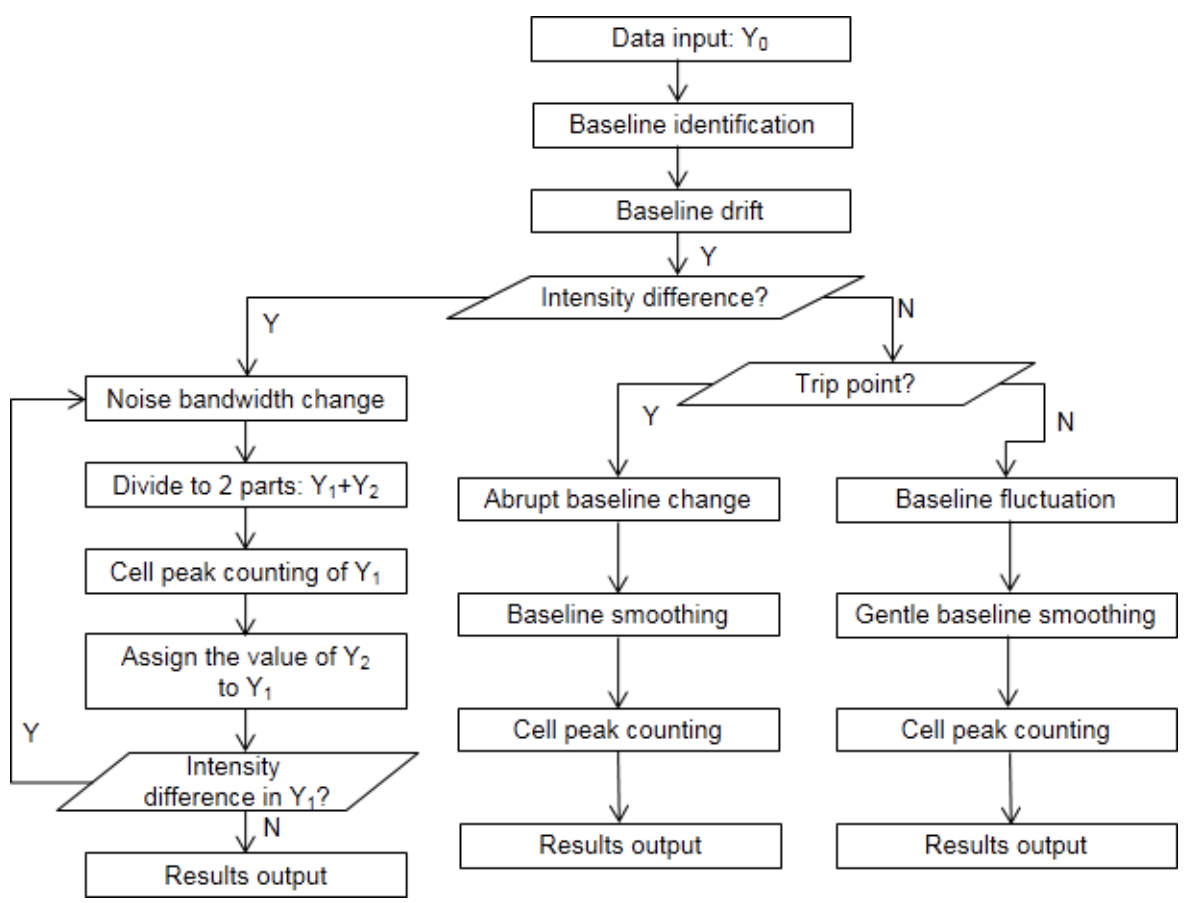

Fig. 3. Flow chart of the processing method. Concrete operations of baseline correction method are described as follows: using the interpolation fitting method to get a curve that is denoted as baseline level. By subtracting the original signal with the baseline level, we can get the corrected signal with a less fluctuating baseline level. Check if there is any trip point in the corrected signal, and then check if there is intensity difference between both sides of the trip point. Count cell peaks according to the formula and output results both in figure and text form.

better than cubic spline interpolation. After fitting these sampling points, an estimated baseline of the original signal is generated. The following procedure is subtracting the signal intensity with the new baseline at every signal point, and those irregular baseline drifts could be eliminated.

\subsubsection{Automated segmenting}

The following step is to search trip points of the baseline, referring to points with significant intensity change. By setting a threshold, the algorithm can automatically check if there is any point whose signal intensity difference of its both sides reaches beyond the threshold, if yes, such a point is defined as a trip point. The reason why we choose to check trip points in the interpolation-fitted baseline rather than in noise band of the original signal is that, in the original signal, the most obvious signal intensity difference is between a cell peak and the noise band, rather than fluctuation in baseline, therefore, it does not conform to the definition of a trip point we mentioned above. By checking trip points in the interpolation-fitted baseline, the algorithm focused on checking signal intensity difference caused by baseline drifting, thus facilitating the following steps.

After searching for trip points, the algorithm goes on to check if the difference of mean signal intensity during a given period of time at both sides of the trip point reaches beyond the setting threshold. If not, the problem of the signal is just baseline drift rather than noise bandwidth change, and baseline drift has already been solved in the first step. If the bandwidth difference reaches beyond the threshold, the signal will be segmented into two parts in such a trip point, which is defined as a segmenting point. The threshold that determines whether or not to segment the signal is a value based on an empirical parameter: $0.2^{*}$ mean $(Y)$, where $Y$ stands for the signal intensity. This empirical value has been tested for a large amount of data and proved to be a reliable value. The threshold depends on the signal intensity, therefore it would change for different data sets. Then the software will process the segmented two parts separately.

Once the algorithm finds a segmenting point, it will separate the signal into two parts and process the first part of the signal individually. However, for 
some signals with severe noise bandwidth change, whether or not there are any other segmenting points in the second part of the signal is not certain. Therefore, a while-loop is introduced in order to find other segmenting points in the second part of the separated signal by further automatic segmenting.

\subsubsection{Wavelet-based denoising and peak picking}

The wavelet-based denoising processing method has been reported previously. ${ }^{32}$ In our work, Symlet 6 was used as mother wavelets, and the wavelet decomposition scale was 3. Procedure following wavelet denoising is peak picking. The peak is defined as local maximum of the signal intensity, and one peak corresponds to one detected fluorescencelabeled cell. The threshold is determined by the following formula:

$$
\text { Threshold }=\text { Median }+ \text { Multiplier } * \text { MAD/0.6745, }
$$

where "median" is the median value of the signal intensity, which is more reasonable than mean value; the "multiplier" is a correction coefficient that determines the rigor level of the threshold, whose value is usually set as seven for general IVFC signals. Further detailed information about multiplier was illustrated in our previous work. ${ }^{24}$ "MAD" is the abbreviation for the median absolute deviation of all the signal intensities, reflecting the degree of variation. This formula is based on the theory that the baseline level of IVFC signals conforms to a Gaussian distribution, thus fluorescently-labeled peak signals are viewed as abnormal values. In practice, if the signal intensity reaches beyond the threshold, it would be counted as a peak candidate. Every peak candidate would be examined further to remove the overlapped peaks or the false positive peaks whose peak widths are too narrow, which are generally caused by the electrical noise.

\subsubsection{Metrics}

We inspected cell peak numbers using Measure Foundry software as an assistant viewing tool. The manually counted cell numbers were seen as true value. Then, we compared the efficacy by calculating Pearson's correlation coefficient and the meansquare error (MSE) between the true value and cell numbers obtained by correcting baseline drift and by the previous dynamic peak picking method.

\section{Results}

\subsection{The baseline drift was removed in signals with noise band width change}

The signal shown in Fig. 4(a) was a representative signal with noise bandwidth change, where the bandwidth decreased dramatically since $57 \mathrm{~s}$. If we adopt the previous dynamic peak picking method (without baseline correction), the result would be highly inaccurate. The evident noise bandwidth change would influence the accuracy of auto-determination of threshold by the formula mentioned in Eq. (1), and consequently, influence the accuracy of peak picking: thousands of signals would be calculated as positive peaks within the first $57 \mathrm{~s}$, while almost no signal would be regarded as peaks from $57 \mathrm{~s}$ to the end (shown in Fig. S1). The original signal shown in Fig. 4(a) was divided into two parts, and the circles in Figs. 4(c) and 4(d) represented recognized cells. Graphical results showed that there were eight cell peaks in the first $57 \mathrm{~s}$, instead of thousands of peaks. In the output text file which recorded cell numbers in every minute, the cell numbers in the first $60 \mathrm{~s}$ were eight, which was verified by manually counting numbers. The sensitivity was improved greatly from $35.71 \%$ by the dynamic peak picking method to $85.71 \%$, and the specificity was improved from $99.93 \%$ to $99.99 \%$. For signals without baseline drift, the dynamic peak picking method can count cell numbers with a high accuracy. The reason why it performed with a low sensitivity is that we have chosen the signal with severe baseline drift, in order to illustrate the importance of baseline correction. The formula used to count sensitivity and specificity in this work is described in the supplementary material.

\subsection{Cell counting results became more accurate in signals with gentle baseline fluctuation}

In a similar way, gentle baseline fluctuation caused by animal's respiration would influence the determination of the threshold (Fig. 5). By using the previous dynamic peak picking method, errors 


\section{Wang et al.}

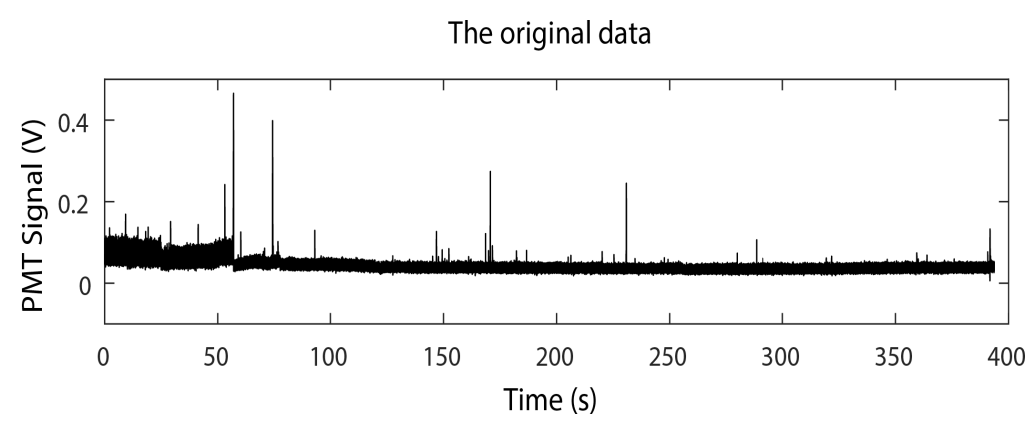

(a)

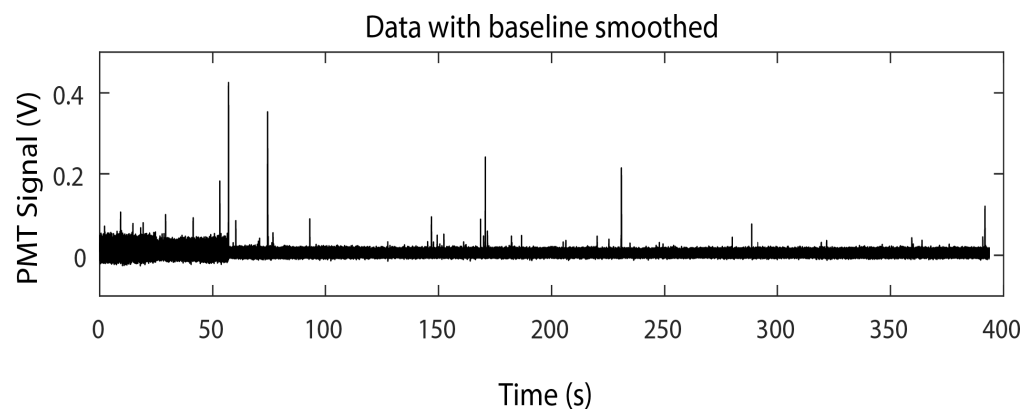

(b)

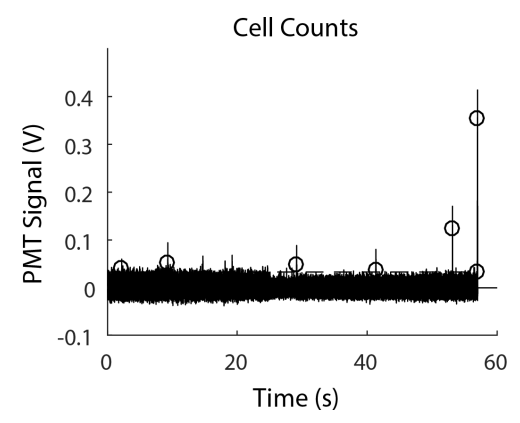

(c)

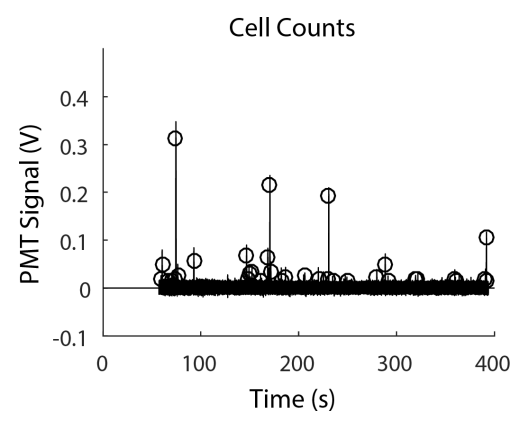

(d)

Fig. 4. A signal with baseline intensity change is divided into two parts and is processed separately. (a) The original signal has an obvious baseline intensity difference since the end of the first minute. (b) The baseline of signal is less fluctuated after baseline smoothing. (c) Cell peak counting result of the first signal part shows there are eight cells in the first minute. (d) The second part of the signal after auto-segmentation shows corresponding cell counting result (Table 1).

would be in a tolerable range since the fluctuation level was not severe. However, this new processing method could realize the peak picking procedure with higher accuracy. For example, some peaks with low intensity might be omitted by previous methods. The dash line in Fig. 5(c) represented the calculated threshold. To verify the accuracy of this method, the results were compared with manual

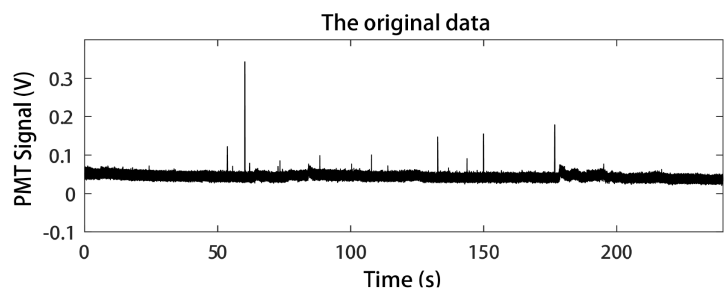

(a)

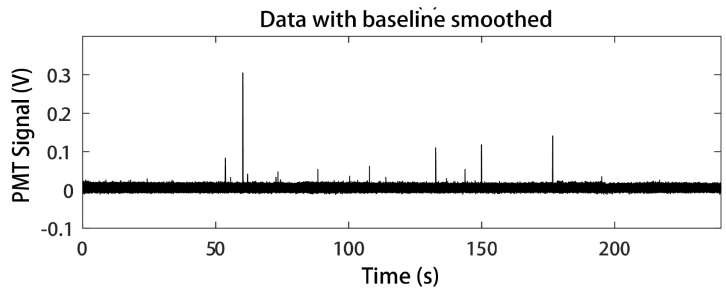

(b)

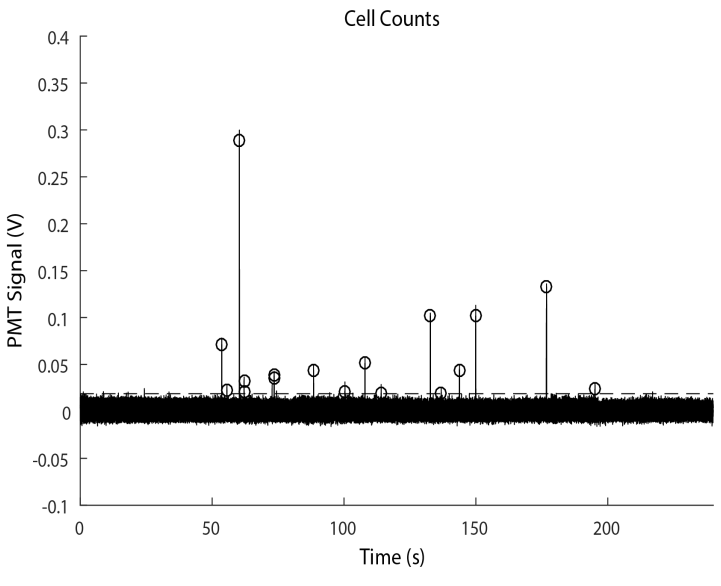

(c)

Fig. 5. A signal with gentle baseline fluctuation is processed after correcting baseline drift. (a) The baseline level of the original signal fluctuated irregularly. (b) The baseline of the signal is less fluctuated after correcting the drift. (c) Cell peaks after correcting baseline drift shows the counting result of cells (Table 2). 
Table 1. Cell count results for a signal with baseline intensity change.

\begin{tabular}{lccccccc}
\hline Time (min) & $\begin{array}{c}\text { Average cell } \\
\text { counts }\end{array}$ & 1 & 2 & 3 & 4 & 5 & 6 \\
\hline $\begin{array}{c}\text { Without baseline } \\
\text { correction }\end{array}$ & 237.3 & 1418.0 & 2.0 & 2.0 & 1.0 & 1.0 & 0.0 \\
$\begin{array}{c}\text { With baseline } \\
\text { correction }\end{array}$ & 6.7 & 8.0 & 5.0 & 9.0 & 8.0 & 6.0 & 4.0 \\
\hline
\end{tabular}

Table 2. Cell count results for a signal with gentle baseline fluctuation.

\begin{tabular}{lccccc}
\hline Time (min) & Average cell counts & 1 & 2 & 3 & 4 \\
\hline $\begin{array}{c}\text { Without baseline } \\
\text { correction }\end{array}$ & 13.8 & 3.0 & 9.0 & 42.0 & 1.0 \\
$\begin{array}{c}\text { With baseline } \\
\text { correction }\end{array}$ & 4.3 & 5.0 & 6.0 & 5.0 & 1.0 \\
\hline
\end{tabular}

Table 3. Cell count results for a signal with abrupt baseline level change.

\begin{tabular}{|c|c|c|c|c|c|c|c|c|c|c|c|}
\hline Time (min) & Average cell counts & 1 & 2 & 3 & 4 & 5 & 6 & 7 & 8 & 9 & 10 \\
\hline Without baseline correction & 0.1 & 0.0 & 0.0 & 0.0 & 0.0 & 0.0 & 1.0 & 0.0 & 0.0 & 0.0 & 0.0 \\
\hline With baseline correction & 14.5 & 12.0 & 7.0 & 27.0 & 20.0 & 12.0 & 19.0 & 21.0 & 7.0 & 12.0 & 8.0 \\
\hline
\end{tabular}

counting results, which were 17 and 19 cells, separately (Table 3). For this signal, the sensitivity was improved from $84.21 \%$ by the dynamic peak picking method to $89.47 \%$.

\subsection{Detecting sensitivity increased greatly in signals with abrupt baseline level change}

Because of experimental animal's sudden movement during data recording, the problem of abrupt baseline level change exists in many IVFC signals (Fig. 6(a)). If we analyze those flawed signals using the dynamic peak picking algorithm, threshold calculated by average baseline level would be highly inaccurate: baseline level that dramatically drifted up and down could make the accurate determination of threshold nearly impossible (shown in Fig. S2). The inaccurate threshold would further influence cell peak counting. However, by using this method, the overall baseline could be dragged to the same level (Fig. 6(b)). Results of the calculated threshold and the following peak picking procedure were reliable (Fig. 6(c)), conforming to the manually counted cell numbers which represented standard results. The sensitivity was improved significantly from $0.62 \%$ by the dynamic peak picking method to $90.06 \%$.

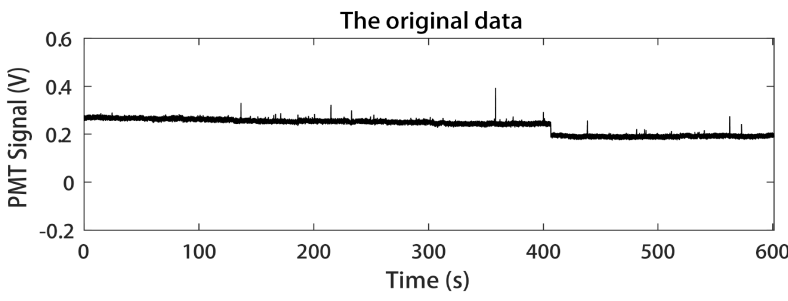

(a)

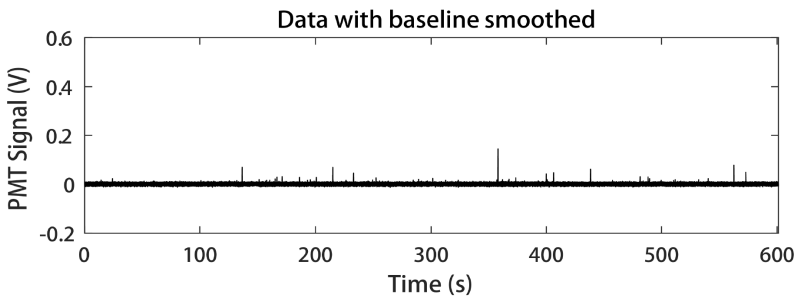

(b)

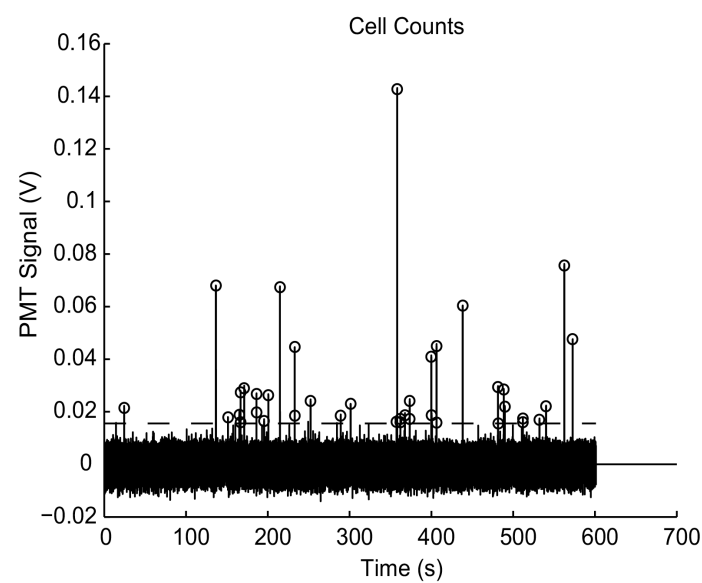

(c)

Fig. 6. A signal with abrupt baseline level change is processed after correcting baseline drift. (a) The baseline level of the original signal changes at around $406 \mathrm{~s}$. (b) The smoothed signal has a less fluctuating baseline level. (c) Cell peaks after correcting baseline drift shows the counting result of cells. 


\subsection{Verifying the accuracy}

To verify the accuracy of our method, the Pearson correlation coefficient $R^{2}$ and the MSE were calculated by testing 23 representative IVFC signals with baseline drift. $R^{2}$ for the baseline correcting method showed a high correlation with true value $\left(R^{2}=0.996, p<0.01\right)$, compared with previous dynamic peak picking method which did not correct baseline drift (correlation coefficient $=-0.121$, not relevant). The MSE obtained for baseline correcting method was significantly lower $(\mathrm{MSE}=1.452)$ than the dynamic peak picking method $(\mathrm{MSE}=29.588)$.

\section{Discussion}

As the IVFC is broadening, its application in various biomedical research areas, baseline drift in signals has become a common problem. In this study, we introduced a new approach that could reduce the influence of baseline drift in the IVFC signals by automatically smoothing the baseline drift, segmenting and analyzing the signal separately. Developed and realized under MATLAB graphical user interface (GUI) platform, this algorithm could effectively solve most of the baseline drift problems relating to noise bandwidth and baseline level.

The advantage of this processing method is that, compared to the line-gating method or the dynamic peak picking method, this method could detect baseline's trip points automatically, divide the signal into parts accordingly and analyze them separately. As the baseline level changes greatly in some signals, previous processing methods may not be able to identify cell peaks effectively by waveletbased denoising. Such problems could be avoided if the baseline has been smoothed and the signal is processed individually. This method enhances the availability of IVFC signals for further analysis, such as counting cell peaks, calculating blood flow velocity, etc. In-house software which integrates this algorithm and other related functions is now in use to facilitate researchers.

In Eq. (1) which defines the threshold for peak identification, the multiplier value is set as $7 \mathrm{usu}-$ ally, which is an empirical value. After being processed by this baseline correcting method, the signal intensity difference between neighboring sampling points gets decreased, thus the MAD factor diminishes correspondingly. According to Eq. (1), the diminished MAD factor minifies the cell peak threshold, leading to inaccuracy such as counting noise peaks as valid cell peaks. By testing and verifying the accuracy, the number of different cell peaks between this processing method and true value was about $4-5$ peaks per minute. One practical solution is to raise the multiplier value from the original 7 to the current 10, which means that since the processing method has been improved, the formula's corresponding parameter also needs to be improved.

\section{Acknowledgments}

This work was supported by the grants of the National Major Scientific Research Program of China (Grant Nos. 2011CB910404 and 2012CB966801), the National Nature Science Foundation of China (Grant No. 61227017) and the National Science Fund for Distinguished Young Scholars (Grant No. 61425006).

\section{References}

1. E. I. Galanzha, M. G. Viegas, T. I. Malinsky, A. V. Melerzanov, M. A. Juratli, M. Sarimollaoglu, D. A. Nedosekin, V. P. Zharov, "In vivo acoustic and photoacoustic focusing of circulating cells," Sci. Rep. 6, 21531 (2016).

2. E. I. Galanzha, E. V. Shashkov, P. M. Spring, J. Y. Suen, V. P. Zharov, "In vivo, noninvasive, label-free detection and eradication of circulating metastatic melanoma cells using two-color photoacoustic flow cytometry with a diode laser," Cancer Res. 69, 7926-7934 (2009).

3. Z. Fan, X. Wei, "In vivo flow cytometry: A powerful optical technology to detect circulating tumor cells and diagnose cancer metastasis in vivo/In-vivoDurchflusszytometrie: Ein leistungsstarkes optisches Verfahren zur Detektion zirkulierender Tumorzellen und zur In-vivo-Diagnose von Metastasen," Photonics Lasers Med. 2, 27-35 (2013).

4. J. Novak, I. Georgakoudi, X. Wei, A. Prossin, C. Lin, "In vivo flow cytometer for real-time detection and quantification of circulating cells," Opt. Lett. 29, 77-79 (2004).

5. V. P. Zharov, E. I. Galanzha, V. V. Tuchin, "Photothermal image flow cytometry in vivo," Optics Lett. 30, 628-630 (2005).

6. V. P. Zharov, E. I. Galanzha, E. V. Shashkov, N. G. Khlebtsov, V. V. Tuchin, "In vivo photoacoustic flow cytometry for monitoring of circulating single 
cancer cells and contrast agents," Opt. Lett. 31, 3623-3625 (2006).

7. Y. Li, Z. Fan, J. Guo, G. Liu, X. Tan, C. Wang, Z. Gu, X. Wei, "Circulation times of hepatocellular carcinoma cells by in vivo flow cytometry," Chin. Opt. Lett. 8, 953-956 (2010).

8. J. Yan, Z. Fan, X. Wu, M. Xu, J. Jiang, C. Tan, W. Wu, X. Wei, J. Zhou, "Circulating tumor cells are correlated with disease progression and treatment response in an orthotopic hepatocellular carcinoma model," Cytometry A 87, 1020-1028 (2015).

9. I. Georgakoudi, N. Solban, J. Novak, W. L. Rice, X. Wei, T. Hasan, C. P. Lin, "In vivo flow cytometry a new method for enumerating circulating cancer cells," Cancer Res. 64, 5044-5047 (2004).

10. D. A. Sipkins, X. Wei, J. W. Wu, J. M. Runnels, D. Côté, T. K. Means, A. D. Luster, D. T. Scadden, C. P. Lin, "In vivo imaging of specialized bone marrow endothelial microdomains for tumor engraftment," Nature 435, 969-973 (2005).

11. Z.-C. Fan, J. Yan, G.-D. Liu, X.-Y. Tan, X.-F. Weng, W.-Z. Wu, J. Zhou, X.-B. Wei, "Real-time monitoring of rare circulating hepatocellular carcinoma cells in an orthotopic model by in vivo flow cytometry assesses resection on metastasis," Cancer Res. 72, 2683-2691 (2012).

12. X. Wei, D. A. Sipkins, C. M. Pitsillides, J. Novak, I. Georgakoudi, C. P. Lin, "Real-time detection of circulating apoptotic cells by in vivo flow cytometry," Mol. Imaging 4, 415 (2005).

13. Z. Fan, J. A. Spencer, Y. Lu, C. M. Pitsillides, G. Singh, P. Kim, S. H. Yun, V. Toxavidis, T. B. Strom, C. P. Lin, "In vivo tracking of 'color-coded' effector, natural and induced regulatory $\mathrm{T}$ cells in the allograft response," Nat. Med. 16, 718-722 (2010).

14. J. Guo, Z. Fan, Z. Gu, X. Wei, "Studying the role of macrophages in circulating prostate cancer cells by in vivo flow cytometry," J. Innov. Opt. Health Sci. 5, 1250027 (2012).

15. Y. A. Menyaev, D. A. Nedosekin, M. Sarimollaoglu, M. A. Juratli, E. I. Galanzha, V. V. Tuchin, V. P. Zharov, "Optical clearing in photoacoustic flow cytometry," Biomed. Opt. Exp. 4, 3030-3041 (2013).

16. S. Lee, C. Vinegoni, P. F. Feruglio, L. Fexon, R. Gorbatov, M. Pivoravov, A. Sbarbati, M. Nahrendorf, R. Weissleder, "Real-time in vivo imaging of the beating mouse heart at microscopic resolution," Nat. Commun. 3, 1054 (2012).

17. M.-C. Zhong, X.-B. Wei, J.-H. Zhou, Z.-Q. Wang, Y.M. Li, "Trapping red blood cells in living animals using optical tweezers," Nat. Commun. 4, 1768 (2013).

18. Z. A. Nima, M. Mahmood, Y. Xu, T. Mustafa, F. Watanabe, D. A. Nedosekin, M. A. Juratli, T. Fahmi, E. I. Galanzha, J. P. Nolan, "Circulating tumor cell identification by functionalized silver-gold nanorods with multicolor, super-enhanced SERS and photothermal resonances," Sci. Rep. 4, 4752 (2014).

19. M. V. Khodakovskaya, K. de Silva, D. A. Nedosekin, E. Dervishi, A. S. Biris, E. V. Shashkov, E. I. Galanzha, V. P. Zharov, "Complex genetic, photothermal, and photoacoustic analysis of nanoparticleplant interactions," Proc. Natl. Acad. Sci. 108, 1028-1033 (2011).

20. J.-W. Kim, E. I. Galanzha, E. V. Shashkov, H.-M. Moon, V. P. Zharov, "Golden carbon nanotubes as multimodal photoacoustic and photothermal highcontrast molecular agents," Nat. Nanotechnol. 4, 688-694 (2009).

21. J. Shao, R. J. Griffin, E. I. Galanzha, J.-W. Kim, N. Koonce, J. Webber, T. Mustafa, A. S. Biris, D. A. Nedosekin, V. P. Zharov, "Photothermal nanodrugs: Potential of TNF-gold nanospheres for cancer theranostics," Sci. Rep. 3, 1293 (2013).

22. E. I. Galanzha, E. V. Shashkov, T. Kelly, J.-W. Kim, L. Yang, V. P. Zharov, "In vivo magnetic enrichment and multiplex photoacoustic detection of circulating tumor cells," Nat. Nanotechnol. 4, 855-860 (2009).

23. D. Damm, C. Wang, X. Wei, A. Mosig, "Cell counting for in vivo flow cytometer signals using wavelet-based dynamic peak picking," IEEE 2009 2nd Int. Conf. on Biomedical Engineering and Informatics, pp. 1-4, IEEE, Washington, D.C. (2009).

24. Y. Suo, T. Liu, C. Xie, D. Wei, X. Tan, L. Wu, X. Wang, H. He, G. Shi, X. Wei, "Near infrared in vivo flow cytometry for tracking fluorescent circulating cells," Cytometry A 87, 878-884 (2015).

25. Y. Ding, J. Wang, Z. Fan, D. Wei, R. Shi, Q. Luo, D. Zhu, X. Wei, "Signal and depth enhancement for in vivo flow cytometer measurement of ear skin by optical clearing agents," Biomed. Opt. Exp. 4, 25182526 (2013).

26. Y. Li, J. Guo, C. Wang, Z. Fan, G. Liu, C. Wang, Z. Gu, D. Damm, A. Mosig, X. Wei, "Circulation times of prostate cancer and hepatocellular carcinoma cells by in vivo flow cytometry," Cytometry A 79, 848-854 (2011).

27. C. D. McManus, U. Teppner, D. Neubert, S. M. Lobodzinski, "Estimation and removal of baseline drift in the electrocardiogram," Comput. Biomed. Res. 18, 1-9 (1985).

28. D. Ruan, J. Fessler, J. Balter, P. Keall, "Real-time profiling of respiratory motion: Baseline drift, frequency variation and fundamental pattern change," Phys. Med. Biol. 54, 4777 (2009).

29. V. S. Chouhan, S. S. Mehta, "Total removal of baseline drift from ECG signal," Computing: Theory and Applications, 200\%. ICCTA'0\%. Int. Conf., pp. 512-515, IEEE, USA (2007). 
X. Wang et al.

30. R. F. Von Borries, J. H. Pierluissi, H. Nazeran, "Wavelet transform-based ECG baseline drift removal for body surface potential mapping," 2005 IEEE Engineering in Medicine and Biology. 27th Ann. Conf., pp. 3891-3894, IEEE, USA (2006).
31. L. Xu, D. D. Zhang, K. Wang, "Wavelet-based cascaded adaptive filter for removing baseline drift in pulse waveforms," IEEE Trans. Biomed. Eng. 52, 1973-1975 (2005).

32. D. L. Donoho, "De-noising by soft-thresholding," IEEE Trans. Inf. Theory 41, 613-627 (1995). 